\title{
ESTADO DE LAS PRÁCTICAS DE LECTURA EN LA UNIVERSIDAD COLOMBIANA. UNA APROXIMACIÓN ESTADÍSTICA
}

\section{STATE OF READING PRACTICES IN COLOMBIAN UNIVERSITIES A STATISTICAL APPROACH}

\author{
Juan David Zambrano Valencia* \\ Rubiel Medina Quintero** \\ Beyddy Muñoz Loaiza***
}

Zambrano V. Juan D., Medina Q. Rubiel. Muñoz L. Beyddy. Sophia N 7 - 2011. ISSN: 194-8932 Págs. 39-47.

Recepción: Junio 13 de 2011

Aceptación: Julio 30 de 2011

\section{RESUMEN}

Este artículo presenta el análisis de los resultados de algunas preguntas de la encuesta aplicada a estudiantes de educación superior desde la investigación ¿Para qué se lee y se escribe en la universidad colombiana? Un aporte a la consolidación de la cultura académica del país, con el código PREO0439015708, desarrollado por 16 universidades nacionales. Las observaciones expuestas pertenecen a 4 interrogantes relacionados específicamente con las prácticas de lectura académica con las que se enfrentan los estudiantes durante el transcurso de su pregrado.

\section{PALABRAS CLAVE}

Lectura, estudiantes, profesores, universidades colombianas, encuestas, resultados.

\section{ABSTRACT}

This article presents the analysis of the results from the questions made in recent poll applied to university students from the research "what's the reason to read and write in Colombian universities" an input to the consolidation of the academic

* Licenciado en español y literatura de la Universidad del Quindío. Especialista en enseñanza de la Literatura, maestría en cursos sobre ciencias de la educación, profesor del programa de licenciatura en español y literatura de la Facultad de Educación de la Universidad del Quindío, integrante de la Red de Lenguaje, Eje Cafetero, integrante del Grupo de investigación en Didáctica de la Lengua Materna y la Literatura (DiLeMa). boreasdvn@yahoo.com - Colombia.

** Licenciado en español y literatura de la Universidad del Quindío, especialista en enseñanza de la literatura, estudiante de la maestría en Ciencias de la Educación, integrante de la Red de Lenguaje del Eje Cafetero, profesor del programa de licenciatura en español y literatura de la Facultad de Educación de la Universidad del Quindío. planetarioxd@gmail.com - Colombia.

*** Licenciada en español y iiteratura de la Universidad del Quindío, estudiante de la Maestría en Ciencias de la Educación con Énfasis en Didáctica de la Lengua Materna y la Literatura, Integrante de la Red de Lenguaje, Eje Cafetero, profesora del Programa de Licenciatura en Español y Literatura de la Facultad de Educación de la Universidad del Quindío. beyddy@gmail.com Carrera 15 calle 12 Norte, Armenia, Quindío, Colombia. 
culture in the country, coded PREOO439015708, developed by 16 universities nationwide. The given observations obey to four questionings specifically related to the academic reading practices faced by students during the course of undergraduate.

\section{KEY WORDS}

Reading, students, teachers, Colombian universities, survey, researches.

\section{INTRODUCCIÓN}

La relevancia de la lectura y la escritura en la universidad es indiscutible. Formarse en esta consiste, en gran parte, en estar en condiciones de comprender, interpretar y producir textos propios de los dominios disciplinares y profesionales específicos; es decir, leer y escribir en una disciplina determinada. Por ello Colciencias aprueba en el 2008 la investigación ¿Para qué se lee y se escribe en la universidad colombiana? Un aporte a la consolidación de la cultura académica del país, con el código PREOO439015708, en la cual participan 16 universidades de Colombia ${ }^{1}$, entre ellas la Universidad del Quindío (con código 483 de la Vicerrectoría de Investigaciones de esta casa de estudio), con el grupo de investigación DiLeMa². La citada investigación se enmarca en los siguientes presupuestos:

[...] la universidad promueve ciertos modos de leer y escribir, ciertas prácticas de lectura y escritura, en atención a un tipo de cultura académica que intenta favorecer. En este sentido, detrás de dichas prácticas es posible leer la idea de cultura académica que circula en nuestro país. Esas prácticas, además de estar determinadas por las capacidades lectoras y escritoras de los estudiantes, de sus características socioculturales y sus trayectorias académicas, están marcadas de modo fuerte por elementos como ¿qué se pide leer al estudiante?, ¿para qué se pide leer y escribir?, ¿qué se hace con lo que se lee y se escribe?, ¿cuáles son los mecanismos de legitimación, valoración y evaluación de los productos

1 Pontificia Universidad Javeriana, Universidad del Valle, Universidad del Quindío, Unidad Central Valle del Cauca, Universidad del Pacífico, Universidad Pedagógica Nacional, Universidad de Ibagué, Fundación Universitaria Monserrate, Universidad del Cauca, Universidad de Córdoba, Universidad Autónoma de Occidente, Universidad Católica Popular del Risaralda, Universidad de La Amazonia, Universidad de Antioquia, Universidad del Atlántico, Universidad de Caldas.

2 Grupo de investigación en Didáctica de la Lengua Materna y la Literatura (DiLeMa), aprobado por Colciencias en categoría C, del programa de Licenciatura en Español y Literatura de la Universidad del Quindío. de lectura y escritura en la universidad?, ¿qué clase de apoyos reciben los estudiantes antes, durante y después de la lectura y escritura de textos?

Desde esta problemática se plantea la siguiente hipótesis: los modos de leer y escribir de los estudiantes son un efecto de las condiciones pedagógicas y didácticas que la universidad promueve, así como del tipo de demandas que plantea. Dichos modos de leer y escribir están determinados por otros factores de orden sociocultural que es necesario reconocer. Esta hipótesis permite explorar la idea de cultura académica que subyace a las prácticas de lectura y escritura en la universidad, es decir, caracterizar la cultura académica, desde las prácticas de lectura y escritura, con el fin de derivar orientaciones para el fortalecimiento de dicha cultura en la universidad colombiana (Pérez y Rincón, 2008: 38).

En este orden de ideas, el presente proyecto se vale especialmente de descripción e interpretación y está basado en información documental y datos de naturaleza cuantitativa y cualitativa. La población está constituida por docentes y estudiantes universitarios de pregrado que hacen parte de diferentes programas académicos ofrecidos por un grupo de universidades del país con variadas características: diversidad de regiones, tipo de universidades (privadas, públicas), tamaño de las mismas, entre otras.

En tal contexto se encuestaron 3719 estudiantes de las universidades participantes como parte del proceso metodológico de la investigación, a fin de tener una primera aproximación a las prácticas de lectura y escritura académicas en la universidad colombiana ${ }^{3}$. La encuesta se estructuró en cinco partes. Dos centradas en la lectura y la escritura en general. Allí se le preguntó al grupo de encuestados por las experiencias genéricas de lectura y escritura en su proceso de formación

3 La denominación "universidad colombiana" contempla las
universidades participantes en la investigación. 
profesional, y sobre las mismas prácticas en la universidad. Una tercera parte interrogó por docentes específicos en cuyos cursos se hubiesen desarrollado prácticas destacadas de lectura y escritura. Las partes restantes de la encuesta les preguntaron a los alumnos por particularidades inherentes a esas experiencias concretas de lectura y escritura.

En este artículo se fijará la atención en uno de los cinco segmentos de las encuestas: "la lectura en la universidad", con el propósito de describir y analizar los datos obtenidos y ofrecerle al lector una idea del estado actual de las prácticas de lectura en la universidad colombiana.

\section{FASES METODOLÓGICAS}

El estudio se organizó en seis fases: Fase 0, de validación de la metodología a través del juicio de pares académicos internacionales (Anna Camps, Paula Carlino y Luiz Percival Leme Britto). Fase I, de recolección de información y primer análisis. Fase II, de identificación y caracterización de "prácticas destacadas". Fase III, de conformación de grupos de discusión y entrevistas en profundidad. Fase IV, de elección y sistematización de algunos casos de "prácticas destacadas". Fase V, de elaboración de directrices de política de lectura y escritura para la universidad colombiana. Hasta el momento se han desarrollado las Fases 0, I, II, III y IV.

\section{OBJETIVOS Y PREGUNTAS DE LA INVESTI- GACIÓN}

El objetivo general es "describir, caracterizar, analizar e interpretar las prácticas de lectura y escritura académicas en la universidad colombiana, con el fin de proponer unas orientaciones de política al respecto" (Pérez y Rincón, 2008: 66). Los objetivos específicos son: 1) definir una perspectiva teórica y una matriz de criterios para el análisis de las prácticas de enseñanza de la lectura y la escritura académicas en la universidad, en relación particular con configuraciones didácticas; 2) identificar, categorizar, analizar e interpretar las prácticas de lectura y escritura académicas existentes en la universidad colombiana; 3) reconstruir, describir e interpretar algunas prácticas relevantes ("prácticas destacadas"); 4) producir unas directrices de política de lectura y escritura para la universidad colombiana, de cara a promover una cultura académica que aporte al desarrollo científico del país; 5) realizar aportes para la consolidación de una cultura académica en la universidad colombiana.

Dichas fases y objetivos pretenden responder a las siguientes preguntas: ¿cuáles son las prácticas académicas de lectura y escritura en la universidad colombiana y cómo se caracterizan?, ¿cuáles de estas prácticas son valoradas positivamente por los estudiantes y los docentes?, ¿cómo se explica la presencia de esas prácticas en algunos contextos universitarios? Estos interrogantes subsumen, en relación con las prácticas de enseñanza, otros como: ¿qué se pide leer al estudiante?, ¿para qué se pide leer y escribir?, ¿qué se hace con lo que se lee y se escribe?, ¿cuáles son los mecanismos de legitimación, valoración y evaluación de los productos de lectura y escritura en la universidad?, ¿qué clase de apoyos reciben los estudiantes antes, durante y después de la lectura y escritura de textos?

\section{DESCRIPCIÓN Y ANÁLISIS DE RESULTADOS DE ENCUESTAS}

Es habitual considerar que la enseñanza de la lectura en la universidad colombiana viene signada por prácticas que le apuestan, entre otros asuntos, a comprender géneros discursivos inherentes a los campos disciplinares, a promover prácticas de lectura de textos especializados dentro y fuera del aula de clases y a responder a las exigencias de una asignatura, a las necesidad de la formación profesional y al goce de dichas prácticas. Según Estanislao Zuleta, leer en la universidad es cardinal si se pretende erigir una cultura académica del trabajo y la producción, diferente a una cultura de la obediencia intelectual:

Que leer es trabajar, quiere decir ante todo que no existe un código común al que hayan sido "traducidas" las significaciones que luego vamos a descifrar. El texto produce su propio código por las relaciones que establece entre sus signos; genera, por decirlo así, un lenguaje interior, en relación de afinidad, contradicción y diferencia con otros "lenguajes". El trabajo consiste entonces en determinar el valor que el texto asigna a cada uno de sus términos, valor que puede estar en contradicción con el que posee el mismo término en otros textos (1994: 102). 
En los escenarios en los cuales se estudia la lengua como medio, uno de los valores vitales de la lectura es aproximar a los estudiantes al conocimiento disciplinar más que a la apropiación del sistema de la lengua o a su uso en diversos contextos (como sí ocurre en los programas de Lengua). Naturalmente aquello sufre variaciones en disciplinas y asignaturas de diferentes programas académicos. De ahí que Camargo, Uribe y Caro (2011: 67) sostengan que la educación superior debe desarrollar habilidades comunicativas y creativas en el estudiantado para que asuma de modo apropiado los retos que supone la sociedad actual; y que el docente tenga la responsabilidad de dinamizar procesos de comprensión y producción textuales, proponer tipos y modos discursivos disímiles, "en los cuales el alumno formule distintas relaciones lógicas, construidas a partir del uso adecuado de la lengua y de un conocimiento racional y crítico de la cultura".

Es posible mencionar, entonces, que los propósitos que deberían sostener las prácticas de lectura se corresponden, entre otros, con los siguientes intereses: dinamizar los procesos de aprendizaje de los contenidos disciplinares, acrecentar la base léxica de los estudiantes y hacerlos partícipes de la vida académica, superar problemas de comprensión de varios niveles, leer el mundo y trascender el aula de clases.

A pesar de lo antedicho, los resultados de las encuestas ponen de relieve una realidad contraria en mucho. Este análisis parte de los datos obtenidos en los primeros ítems relacionados con la lectura; puntualmente en los numerales 1 , 3,5 y 8 de la encuesta. Para comprender mejor esos resultados se aclara que en el momento de aplicar las encuestas los estudiantes tenían la alternativa de marcar varias opciones. Las preguntas fueron:

- (1) ¿Para cuáles de las siguientes actividades académicas usted lee?

- (3) ¿Con qué propósitos lee en las actividades académicas seleccionadas en la pregunta 1?

- (5) Señale los documentos que más leyó en el semestre pasado para responder a sus compromisos académicos.

- (8) ¿En qué otro idioma, diferente al español, leyó documentos completos el semestre pasado?

\section{¿Para qué leen los estudiantes universitarios en Colombia?}

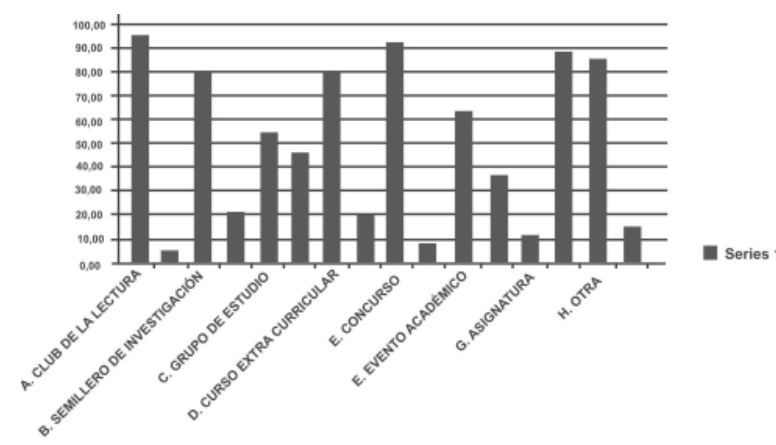

Gráfico 1. Los Autores.

Se observa (pregunta 1, ¿Para cuáles de las siguientes actividades académicas usted lee?) que del número total de estudiantes encuestados (3719), el $88,78 \%$ expresan que "leen para una asignatura", y el $46,73 \%$ afirman que leen mientras participan en un "grupo de estudio". Estos datos discrepan de la opción "el club de la lectura" por la que solo se interesa el $5,43 \%$, siendo este el porcentaje más bajo. Dichos resultados demuestran que los estudiantes universitarios en Colombia, en forma mayoritaria, dirigen sus actividades lectoras hacia las necesidades propias de sus asignaturas y que son propuestas por sus docentes. Sin embargo, si el $88,78 \%$ de esta población lee para su asignatura, suponiendo que esta es la razón de ser de su actividad académica, entonces ¿qué lee el $11.22 \%$ restante de estudiantes cuando cursa sus asignaturas? ¿Esto que leen aporta significativamente al desarrollo de las capacidades comunicativas y creativas de los estudiantes? ¿Dichas lecturas se encuentran comprometidas con el fortalecimiento de la cultura académica?

Preocupa que un bajo número de encuetados lee para "semilleros de investigación" $(20,17 \%)$ y "eventos académicos" (37,46\%). Neus Sanmartí (2010: 145) menciona que leer hace parte de la actividad científica, lo cual invita a quienes trabajan, por ejemplo, en el terreno de la ciencias exactas, a leer lo que han escrito otros científicos sobre los temas que investigan con el doble propósito de conocer el estado del arte del objeto de estudio y contrastar sus nuevas preguntas, datos e idean con otros puntos de vista. Esta opinión tensiona la reducida presencia de lecturas en el marco de semilleros de investigación y eventos académicos; 
actividades que exigen leer con rigor los discursos de una o más disciplinas, interrelacionar enfoques epistemológicos, conocer los campos de aplicación de diversos conocimientos y saber cómo se divulgan los resultados de una investigación.

Llama la atención que casi la mitad de la población lee para un grupo de estudio $(46,73 \%)$. Se infiere que la lectura para este grupo cuenta con un ánimo distinto, ya que el estudiante tiene más autonomía de pertenecer a tales espacios y, por lo tanto, quizás considera que el ejercicio lector está ligado a la construcción de conocimiento, pero no a las exigencias del docente y a un resultado cuantitativo.

En relación con la opción más escogida por los estudiantes ("leen para una asignatura") vale la pena discutir acerca de los siguientes cuestionamientos: ¿las lecturas propuestas por los docentes en sus asignaturas cuentan con orientaciones y acompañamiento?, ¿existe una consigna o guía proporcionada para le lectura de los textos?, ¿se realiza un rastreo de conocimientos previos de los estudiantes antes de iniciar la actividad lectora? Estos interrogantes se plantean dado que, según estudios vigentes (Smith, 1971; Kitsch y van Dijk, 1978; Goodman, 1994; Kintsch, 1994), el docente, al elegir un texto debe tener un propósito definido, saber con exactitud qué se puede hacer con él para que el estudiante no reciba la lectura como "un dejar hacer", lo cual no significa que solo es válida la posición del docente, puesto que, además, la lectura es una fuente de construcción de conocimiento, de diálogo entre el autor y el estudiante, sostenido por los conceptos de su disciplina o profesión.

De la misma manera, cabe preguntar si se hace énfasis solo en el producto (respuesta repetitiva del contenido) o, por el contrario, se favorecen procesos de lectura que buscan la construcción e interpretación de conocimiento, en procura de la inmersión de los estudiantes en sus propios ámbitos disciplinares, para lo cual resulta indispensable ofrecer estrategias de lectura que formen un pensamiento crítico, creativo y práctico.

Reflexiones actuales sobre la didáctica de la legua materna (Camilloni, 2000; Calsamiglia y Tusón, 2001; Camps, 2004; Álvarez, 2005; Bronckart, 2007), apuntalan que los docentes deben contar con estrategias que les permitan intervenir efectivamente en las aulas para respaldar los procesos educativos, resolver problemas de aprendizaje y favorecer la enseñanza de contenidos y, en este caso, los procesos de lectura. Tal vez la carencia de dichas estrategias conduce al profesorado a restringir el tipo de actividad académica para el que leen los estudiantes y los propósitos de lectura. Con el objetivo de superar esta problemática, Cassany (2010: 48) sugiere un abanico de estrategias que beneficiarán las prácticas de lectura interpretativas y sus procesos enseñanza:

Trabaja la interpretación con todo tipo de textos; utiliza textos auténticos; utiliza textos paralelos, opuestos o relacionados; incluye textos multimodales; aprovecha las prácticas vernáculas previas; evita la respuesta única o la corrección convergente; fomenta el diálogo entre el alumnado; pregunta sobre el propósito y el punto de vista del autor; fomenta la relectura y el análisis de los puntos relevantes; ayuda al alumno a relacionar la lectura con su mundo.

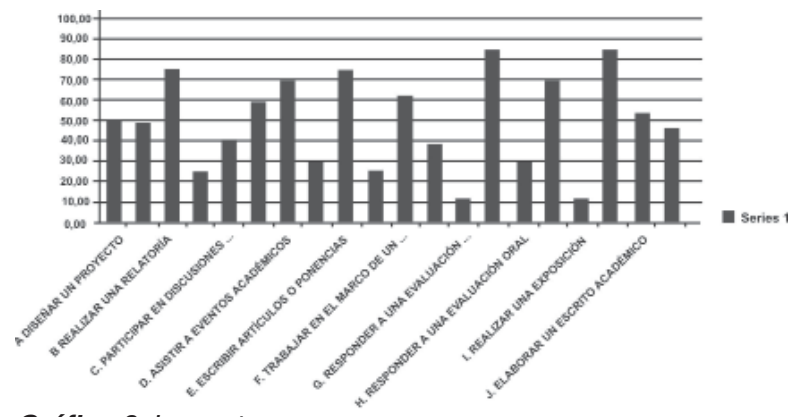

Gráfico 2. Los autores.

En la pregunta 3 (¿Con qué propósitos lee en las actividades académicas seleccionadas en la pregunta 1?), cuyo interés es indagar sobre los propósitos que se traza la población universitaria para leer, el $86,39 \%$ de los estudiantes dijeron que leen para "realizar una exposición", y el 85,99\% para "responder a una evaluación escrita", mientras que únicamente el 24,82\% marcó el ítem "realizar una relatoría", con un porcentaje $(25,84 \%)$ antecedido por "escribir un artículo o ponencia". Las tendencias muestran los puntajes más altos, permiten inferir que una gran población de los jóvenes universitarios leen por la obligación que suponen esas actividades en sus asignaturas. Las exposiciones, por ejemplo, por el hecho de ser públicas, obligan a los estudiantes a que estén mejor preparados para el momento en que 
deban sustentar un tema ante el docente y demás compañeros que, de con frecuencia, sirven como espectadores activos. La exposición implica una lectura obligada tanto del texto expuesto como de otros que lo puedan ampliar.

A propósito de la segunda opción más escogida: "responder a una evaluación escrita" (85,99\%), se puede decir que es congruente con la obligación que sienten los estudiantes por la lectura a fin de obtener una nota que les posibilite aprobar una asignatura.

Inquieta el desinterés de la población encuestada por la lectura de textos que contribuyan a la composición escrita de artículos y ponencias, 25,84\%. Esta inclinación tal vez indica que hay poca motivación por la producción de estos géneros discursivos, y posiblemente se justifique por varias razones: porque no corresponde a la tradición de exigencia de los docentes, porque no tienen el dominio de este tipo de construcción textual, porque existen pocas alternativas de participar en eventos que motiven la publicación en revistas, entre otras.

De cualquier modo, la inmediatez que se observa en las lecturas que hacen los estudiantes para cumplir con sus trabajos académicos y que representan una calificación, deja entrever la poca proyección e interés que quizás pervive en la universidad colombiana para que sus futuros profesionales sean actores y gestores de conocimiento científico.

Leer en la universidad, entonces, implica intervenir en la vida académica; es decir, leer en diversos escenarios (no únicamente en las asignaturas o en los grupos de lectura), priorizar los ejercicios de lectura científica e investigativa, leer con propósitos disímiles (no únicamente para responder una evaluación escrita u oral, realizar un exposición), intensificar los fines de lectura que le apuesten a la participación en eventos académicos y a la escritura científica. También, pensar en prácticas académicas que vean la compresión y producción de textos como procesos correlacionados e interdependientes que prolongan la alfabetización académica y permiten, según Carlino (2005: 22), entender la lectura y la escritura como procesos inherentes a todos los currículos, puesto que componer y comprender textos son medios ineluctables para aprender los contenidos de las disciplinas que conviven en la universidad.

\section{¿Qué leen los estudiantes universitarios en Colombia y en qué idioma?}

La respuesta a tal inquietud está condensada en dos preguntas de la encuesta: señale los documentos que más leyó, en el semestre pasado, para responder a sus compromisos académicos (gráfica 3), y ¿en qué otros idiomas, diferentes al español, leyó documentos completos el semestre pasado? (gráfica 4).

Los estudiantes sostienen que los "apuntes de clase propios" son la fuente fundamental de lectura, $83,68 \%$. No muy lejos de este resultado se encuentran los "materiales elaborados por el profesor", $79,78 \%$; en un tercer lugar están posicionadas las "páginas web y blogs", 76,82\%. Finalmente, entre los textos más leídos, se hallan los "libros o capítulos propios de la carrera", $72,79 \%$.

Los resultados más altos señalan que la fuente fundamental de lectura en la universidad colombiana es lo que se puede encontrar como primeras fuentes; esto es, el aula de clases, los apuntes realizados por los estudiantes y los textos que el docente entrega o

Gráfico 3. Los Autores.

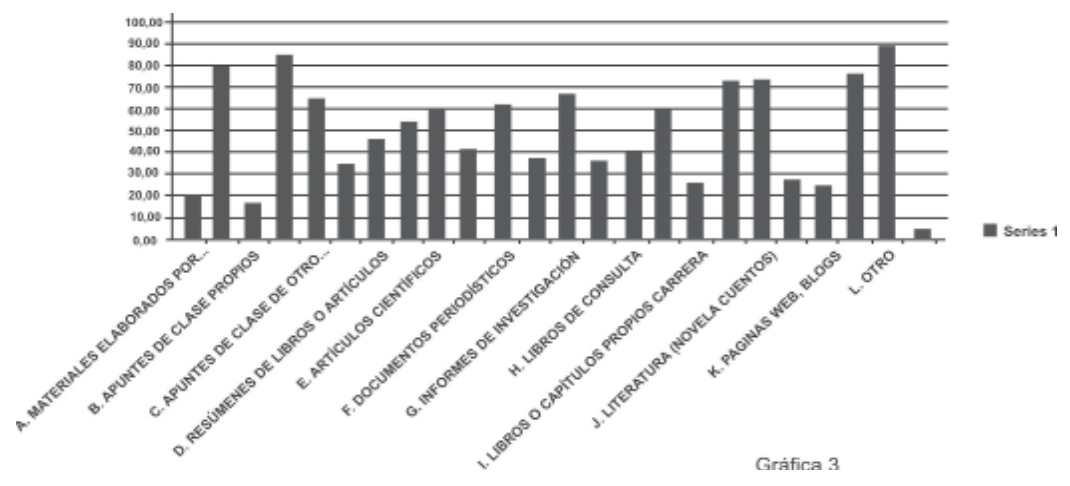


sugiere como bibliografía esencial de la asignatura. Por otro lado, las páginas web, los blogs y los libros relacionados con las temáticas de estudio, son los que más se consultan y se leen en la universidad, situación que no es de desestimar si tenemos en cuenta que las bibliotecas físicas y virtuales -más las segundas- son constantemente visitadas por los estudiantes.

Leer páginas web y blogs pone de manifiesto varios desafíos para los profesores y la universidad; entre ellos, complementar las lecturas tradicionales con lecturas matizadas por hipervínculos, hipertextos, páginas en movimiento; diseñar estrategias que les permitan a los estudiantes afrontar de manera satisfactoria las lecturas virtuales; modernizar las universidades en términos de adquisición, dotación y asimilación de tecnologías vigentes de información y comunicación. En el mismo sentido, Felipe Zayas (2010: 109) expone que leer en internet introduce un significado nuevo del concepto alfabetismo, "que incluye las competencias lectoras tradicionales [...] junto a las nuevas competencias. El hecho de que la lectura en soporte digital esté mediatizada por la tecnología hace que en el actual concepto de alfabetismo se incorporen también los conocimientos tecnológicos necesarios para leer. Pero [...] no se ha de identificar el alfabetismo digital con el conocimiento de las tecnologías".

Otro aspecto relevante de los resultados de la pregunta 5, es el indicador de los textos menos leídos en el aula de clases. Aquí se demuestra lo siguiente: los "artículos científicos" tienen un porcentaje del 40,45 y los "informes de investigación" del 35.74. En consecuencia se pregunta: ¿qué es lo que leen los estudiantes?, ¿qué tipo de información es la que analizan e interpretan? Si bien es sabido que algunos libros y textos son producto de la teoría que fundamenta los conocimientos base de una asignatura, se requiere también de la lectura de investigaciones actuales que demuestren la continuidad de propuestas de antaño o que las refuten. El conocimiento debe ser actualizado y evaluado desde visiones interdisciplinarias y transdisciplinarias, pues se presume que es lo que los estudiantes universitarios necesitan leer.

Desde otra perspectiva, las respuestas a la pregunta ¿En qué otro idioma, diferente al español, leyó documentos completos el semestre pasado? cuestionan la funcionalidad e inclusión de otros idiomas en la universidad colombiana. La respuesta más alta fue inglés, con el $62 \%$.

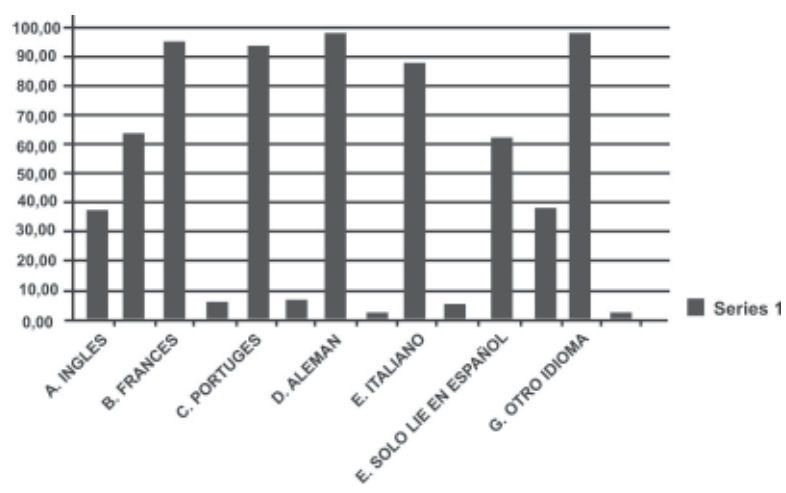

Gráfico 4. Los Autores.

Se sabe que es el segundo idioma más leído por los estudiantes colombianos (62\%) es el inglés, aunque para eso los alumnos deben contar con conocimientos adecuados con el ánimo de comprender lo que los autores desean comunicar. Lo que se intenta manifestar es que las clases de esta asignatura -la de inglés-, deberían tener la mejor calidad y continuidad posible que las instituciones de educación superior le puedan garantizar al alumnado. Tal vez no son suficientes uno o dos semestres de inglés para comprender documentos densos, con tecnicismos y extensión superior a dos páginas. En efecto, ¿qué es lo que se enseña dentro de esta cátedra y cuál es su nivel de profundización?, ¿cuántos semestres provee la universidad colombiana para esto?

Conviene tener en cuenta que el italiano, el francés, el portugués, el alemán, el inglés y otros idiomas son considerados dentro de la bibliografía de los microcurrículos, pues muchos de los autores que allí se refieren están ubicados en países del mismo origen. Con ello en mente, las clases de inglés, por ejemplo, no son suficientes para que los estudiantes puedan tener un buen desempeño en el uso de esta lengua. Los docentes universitarios están destinados al empleo continuo de otros idiomas, por lo menos del inglés, para que los alumnos desarrollen habilidades completas en calidad de profesionales. 


\section{CONCLUSIONES}

De acuerdo con las respuestas referidas, se deduce que la lectura en el aula de clases se orienta de manera unidireccional; en una palabra, con énfasis en la resolución de tareas específicas de determinada asignatura, por lo que vale establecer para qué más se lee en la universidad colombiana y cómo se desarrolla esa práctica académica. De hecho los estudiantes reconocen la importancia de la lectura en cuanto a recepción e indagación de conocimientos; sin embargo: ¿acaso no es también fundamental generar otros espacios de lectura que favorezcan el ejercicio como egresados de calidad? Los resultados abren un interrogante acerca de la trascendencia que puedan tener las prácticas de lectura utilizadas por docentes de algunas universidades del país, en la vida futura profesional y extraacadémica de sus estudiantes. De igual manera, conviene indagar por la presencia en cada Programa de Semilleros suficientes o de otro tipo de grupos que le posibiliten al estudiante potenciar su formación.

Es probable que percibir la lectura (y algunas preguntas relevantes en el proceso lector: qué, por qué y para qué) en tanto práctica propia de cada dominio disciplinar y actividad indispensable de la cultura académica, ofrezca una actitud diferente del profesorado y de sus estudiantes ante dicha actividad y, a la vez, le trace exigencias mayores a los docentes de cara a la cualificación de su formación didáctica y pedagógica: aprender múltiples estrategias de lectura, conocer los múltiples usos de la lectura en la universidad, reconocer y diferenciar las tipologías textuales y géneros discursivos que circulan en el ámbito universitario, orientar procesos de lectura que se inscriban en la producción de textos académicos, enseñar a leer comprensivamente los géneros discursivos propios de un ámbito disciplinar específico, entre otros.

Por esto, siguiendo a Kintsch y van Dijk (1978), consideramos que la lectura de un texto trasluce dos niveles de comprensión. El primero, implica la construcción de la representación proposicional del texto-base (comprender la macro y las microestructuras e identificar la superestructura del texto que se lee). El segundo, supone la construcción de una representación situacional o imagen mental; en este nivel el lector utiliza sus conocimientos previos, plantea inferencias, lee en diversos contextos, adopta una posición crítica frente al texto, ejecuta diversas actividades con base en lo que aprendió de la lectura y asume un papel creativo.

Superar las barreras de la lectura en la universidad, además, exige del profesorado un proceder reflexivo sobre la lectura. Isabel Solé (2010: 17) responde e invita a los docentes a contestar este conjunto de preguntas de aparente fácil resolución: "¿Qué es leer?; ¿cuándo se aprende a leer?; ¿cómo leemos?; ¿es la lectura una cuestión de práctica?; ¿influye la motivación en la lectura?; ¿podemos ayudar a los alumnos a implicarse en la lectura?; ¿podemos ayudar a los estudiantes a ser estratégicos cuando leen?; ¿somos beligerantes a favor de la lectura?".

En este marco, la dificultad expresada por los estudiantes en relación con la lectura de textos académicos, ¿tendrá su origen en que algunos docentes no les ofrecen estrategias adecuadas para guiar y dinamizar esta compleja práctica? o ¿quizás algunos profesores no cuentan con tales estrategias $y$, en efecto, se les dificulta enseñar a leer textos, evitan aproximarse a este ejercicio y eluden, no siempre, la lectura comprensiva propia (interpretar, argumentar y proponer)? o, tal vez, ¿los docentes responsables de la iniciación de los estudiantes universitarios en el mundo de la comprensión textual (piénsese en asignaturas de leguaje básico: Competencias comunicativas, por ejemplo), no cuentan con el tiempo, los espacios y las herramientas suficientes para cumplir de manera óptima con su labor? Lo que sí es cierto, a la luz de los resultados de las encuestas, es que tanto docentes como estudiantes -no todosrequieren y demandan formación estratégica en prácticas de lectura.

Se hace necesario, pues, llevar al salón de clases actividades dirigidas a desarrollar en los estudiantes estrategias cognitivas que les permitan dialogar con distintos tipos de texto, analizar e interpretar, al igual que integrar lo nuevo con lo ya conocido, en una representación mental coherente; y a impulsar estrategias metacognitivas que los conduzcan a asumir el control y la regulación de sus propios procesos de lectura. Con ello en mente, los estudiantes podrán ser lectores independien- 
tes, críticos, que reconozcan su participación en el enriquecimiento de su nivel académico, de manera que el paso por la universidad sea fuente de conocimiento en su vida profesional y personal. Así las cosas, de acuerdo con Carlino, citada por Pérez y Rincón (2008: 51):

Las prácticas de lectura y escritura en el ámbito universitario pueden verse desde el concepto de alfabetización académica, que implica comprender que los modos de leer y escribir son específicos de cada ámbito del saber y que implican un trabajo intencional de parte de los docentes [...] En este sentido coincidimos en que una función fundamental de la universidad es construir modos rigurosos de lectura y escritura propios de las diferentes profesiones y campos disciplinares. De esta forma, es importante señalar que la adquisición de la lengua, la lectura y la escritura no terminan en la universidad. Por el contrario, "la diversidad de temas, tipos de textos, propósitos, destinatarios, reflexión implicada y contextos en los que se lee y escribe plantean siempre en quien se inicia en ellos nuevos desafíos y exigen continuar aprendiendo a leer y escribir" (Carlino, 2002).

\section{REFERENCIAS BIBLIOGRÁFICAS}

Camargo, Zahyra y Uribe, Graciela (2007). "Modelos de comprensión y producción textual y sus posibilidades de proyección didáctica". Cuadernos Interdisciplinarios Pedagógicos, Universidad del Quindío, (8): 25-67.

Camargo, Zahyra; Uribe, Graciela y Caro, Miguel A. (2011). Didáctica de la comprensión y producción de textos académicos. Armenia: OPTIGRAF S.A

Camilloni, Alicia (2000). Corrientes didácticas contemporáneas. Barcelona. Paidós.

Carlino, Paula (2005). Escribir, leer y aprender en la universidad. Una introducción a la alfabetización académica. Buenos Aires: Fondo de Cultura Económica.

García Ferrando, Manuel; Ibáñez, Jesús y Alvira Martín, Francisco (2000). El análisis de la realidad social. Métodos y técnicas de investigación. Madrid. Alianza Editorial.

Cassany, Daniel (2010). "10 claves para enseñar a interpretar". En Ministerio de Educación. Secretaria de Estado de Educación y Formación Profesional (ed.), Con firma 2010. Leer para aprender. Leer en la era digital (pp. 48-53). España.

Kintsch, Walter (1994). "El rol del conocimiento en la comprensión del discurso: un modelo de construcción-integración”. En Textos en contexto II. Los procesos de lectura y escritura ( $\mathrm{pp}$. 69-138). Buenos Aires: Lectura y Vida.
Kintsch, Walter y Dijk, Teun Van (1978). "Towards a model of discourse comprehension and production". Psychology Review, 85: 363-394.

Litwin, Edith (1997). Las configuraciones Didácticas. Una nueva agenda para la enseñanza superior. Buenos Aires: Paidós.

Pérez Abril, Mauricio y Rincón, Gloria (2008). Proyecto: ¿Para qué se lee y se escribe en la universidad colombiana? Un aporte a la consolidación de la cultura académica del país (pp. 34-35). Bogotá: Colciencias.

Sanmartí, Neus (2010). "Leer para aprender ciencias". En Ministerio de Educación. Secretaria de Estado de Educación y Formación Profesional (ed.), Con firma 2010. Leer para aprender. Leer en la era digital (pp. 144-156). España.

Solé, Isabel (2010). "Ocho preguntas en torno a la lectura y ocho respuestas no tan evidentes". En Ministerio de Educación. Secretaria de Estado de Educación y Formación Profesional (ed.), Con firma 2010. Leer para aprender. Leer en la era digital (pp. 17-24). España.

Zayas, Felipe (2010). "Leer en la Red". En Ministerio de Educación. Secretaria de Estado de Educación y Formación Profesional (ed.), Con firma 2010. Leer para aprender. Leer en la era digital (pp. 109-114). España.

Zuleta, Estanislao (1994). "Sobre la lectura". En Elogio de la dificultad y otros ensayos (pp. 101-111). Cali: Fundación Estanislao Zuleta. 